\title{
Synthetic dimensions and topological chiral currents in mesoscopic rings
}

\author{
Hannah M. Price $\odot,{ }^{1}$ Tomoki Ozawa $\odot,{ }^{2,3}$ and Henning Schomerus $\odot^{4}$ \\ ${ }^{1}$ School of Physics and Astronomy, University of Birmingham, Edgbaston, Birmingham B15 2TT, United Kingdom \\ ${ }^{2}$ Advanced Institute for Materials Research, Tohoku University, Sendai 980-8577, Japan \\ ${ }^{3}$ Interdisciplinary Theoretical and Mathematical Sciences Program (iTHEMS), RIKEN, Wako, Saitama 351-0198, Japan \\ ${ }^{4}$ Department of Physics, Lancaster University, Lancaster LA1 4YB, United Kingdom
}

(Received 28 June 2019; revised 28 April 2020; accepted 30 June 2020; published 16 July 2020)

\begin{abstract}
The recently introduced concept of synthetic dimensions allows for the realization of higher-dimensional topological phenomena in lower-dimensional systems. In this paper, we propose a setup where synthetic dimensions arise in mesoscopic hybrid devices and discuss how they provide a natural route to topological states. We demonstrate this for the current induced into a closed one-dimensional Aharonov-Bohm ring by the interaction with a dynamic mesoscopic magnet. The quantization of the magnetic moment provides a synthetic dimension that complements the charge motion around the ring. We present a direct mapping that places the combined ring-magnet system into the class of quantum Hall models and demonstrate that topological features, combined with the magnet's anisotropy, can lead to clear signatures in the persistent current of the single-particle ground state. Our synthetic-dimension model also extends to the many-electron case, where the collective electronic motion couples with the magnet.
\end{abstract}

DOI: 10.1103/PhysRevResearch.2.032017

The recent approach of "synthetic dimensions" opens up routes to realizing topological energy bands in engineered systems of atoms or photons [1,2]. This idea exploits internal states of a system by reinterpreting them as lattice sites along an extra synthetic spatial dimension [3]. Similar to earlier proposals for simulating higher-dimensional models by increasing lattice connectivity [4,5], this provides a way to effectively increase spatial dimensionality; a system with one real spatial and one synthetic dimension can access twodimensional topological phenomena, such as quantum Hall models with topological energy bands and robust chiral edge modes that propagate one way around the system [1].

Since the first theoretical works in cold atoms [1,3], there has been a rapid development of both proposals and experiments to realize different implementations of this concept [2,6-24]. However, so far, this activity has primarily focused on photonic or atomic setups instead of on solid-state electronic systems. Here we show that the concept of a synthetic dimension also emerges naturally in the solid-state context of a mesoscopic ring coupled to a nanomagnet.

The induction of a current around a closed ring by a magnetic flux is an archetypical mesoscopic effect [25]. Such a current can flow even in equilibrium, where the persistent current is a property of the ground state. In normal rings, the current occurs as a mesoscopic effect at very low temperatures, and when measured precisely agrees with the predictions of a simple single-particle picture [26,27]. In this

Published by the American Physical Society under the terms of the Creative Commons Attribution 4.0 International license. Further distribution of this work must maintain attribution to the author(s) and the published article's title, journal citation, and DOI. paper, we study the case where the magnetic flux through the ring is created by a dynamical mesoscopic nanomagnet [28-30]. After introducing the setup, we show that this hybrid system can be understood in a single-particle picture as a synthetic two-dimensional lattice spanned by the position of the electron around the mesoscopic ring together with the magnetic spin states, which serve as a discrete synthetic dimension. The coupling of the electron to the nanomagnet can be reinterpreted as an artificial magnetic field in this lattice, mapping the model to a two-dimensional quantum Hall system. As we show, the mesoscopic ring-magnet system is characterized by topological energy bands and topological "edge" modes, corresponding to spin-polarized chiral currents around the ring. These topological modes can lead to characteristic spin-switching jumps in the ground-state persistent current. As we also discuss, in contrast to previous syntheticdimension proposals, the multielectron case can be reduced to an effective single-particle description capturing collective motion of electrons. These results show that synthetic dimensions are also relevant in the solid state, providing a useful viewpoint that reveals topological effects in settings with potential electronic applications.

Setup. In our hybrid system, a magnetic flux passing through a mesoscopic ring is created by a nanomagnet, represented by a total spin $\mathbf{S}$ with spin quantum number $s$, as shown schematically in Fig. 1(a). The total magnetic flux piercing the ring depends on the magnet's spin state, as labeled by the quantum number $m=-s,-s+1, \cdots, s$. The presence of the flux induces electrons to move around the ring, such that the system is characterized by the spin state, $m$, together with the angular coordinate, $\varphi$, of the moving (spinless) charge.

The key concept of our proposal is to map this hybrid setup to a two-dimensional quantum Hall system on a cylinder, by 

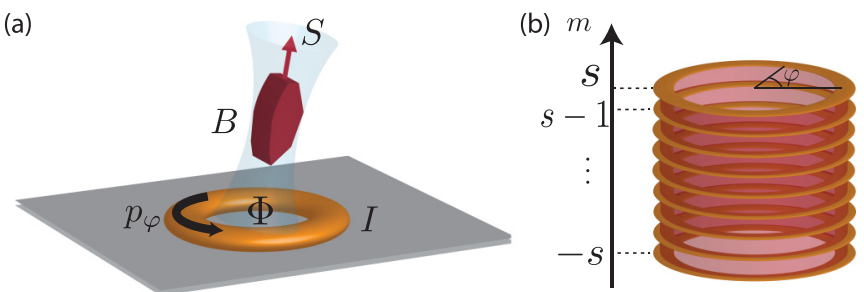

FIG. 1. (a) Sketch of a mesoscopic magnet with spin $\mathbf{S}$ (spin quantum number $s \gg 1$ ) inducing a current $I$ in a mesoscopic closed ring. The current is the response to the magnetic flux $\Phi$ induced by the field $\mathbf{B}$, which couples to the momentum $p_{\varphi}$ of a charge moving around the ring. (b) The situation described in (a) can be mapped to an artificial two-dimensional (cylindrical) lattice indexed by the angular coordinate $\varphi$ of the particle on the ring and the spin quantum number, $m$, of the nanomagnet. The former corresponds to a continuous and periodic (real) spatial dimension, while the latter is a discrete (synthetic) dimension with $2 s+1$ sites.

reinterpreting each spin state $m$ as a different lattice site along a synthetic dimension, as sketched in Fig. 1(b). As we will show, this analogy goes far. The combined magnetoelectronic states form flattened-out bands with nonzero Chern numbers and chiral edge states according to the bulk-boundary correspondence. These chiral edge states have a simple interpretation in terms of a robust mesoscopic spin-orbit locking and leave direct signatures in the persistent current in the ground state of the combined system.

Hamiltonian of the hybrid system. Before illustrating the mapping to a synthetic dimension, we introduce the Hamiltonian of the hybrid ring-magnet system [Fig. 1(a)]. We shall assume that the magnetic moment of the nanomagnet is aligned with its spin so the flux induced into the ring is proportional to the spin operator $S_{z}$, which at the same time is taken to align with one of the principal axes of the magnet's matrix of inertia. The spin quantum numbers $m$ introduced above then correspond to the eigenvalues of $S_{z}$. In a symmetric gauge, the vector potential felt by a charged particle on the ring has $A_{\varphi} \propto \rho S_{z}$ where $\rho$ is the ring radius. In appropriate units, the Hamiltonian for a single electron then takes the simple form

$$
H=\frac{1}{2 M \rho^{2}}\left(-i \nabla_{\varphi}-\gamma S_{z}\right)^{2}+\alpha S_{x}^{2}+\beta S_{y}
$$

where the kinetic-energy term includes the effect of the magnetic flux via minimal coupling with strength $\gamma$. The remaining terms describe the dynamics of the magnet as a rigid body, where $\alpha S_{x}^{2}$ determines its anisotropy while additional quadratic terms can be partially eliminated using the fact that $S_{x}^{2}+S_{y}^{2}+S_{z}^{2}$ is a $c$ number (thus absorbing a constant into the energy). The term $\beta S_{y}$ induces a preferred orientation of the nanomagnet and breaks the full integrability of the system [31].

Mapping to a synthetic dimension. We map the Hamiltonian Eq. (1) to a $2 \mathrm{D}$ quantum Hall system by re-interpreting the eigenstates $|m\rangle$ of $S_{z}$ as different lattice sites along a synthetic dimension [Fig. 1(b)]. In this language, the $S_{x}$ and $S_{y}$ operators lead to "hoppings" along the synthetic dimension, as they can be expressed by ladder operators as $S_{x}=\left(S_{+}+S_{-}\right) / 2$ and

$$
\begin{aligned}
& S_{y}=\left(S_{+}-S_{-}\right) / 2 i \text { with } \\
& S_{+}|m\rangle=\sqrt{s(s+1)-m(m+1)}|m+1\rangle \equiv 2 t_{m}|m+1\rangle, \\
& S_{-}|m\rangle=\sqrt{s(s+1)-m(m-1)}|m-1\rangle=2 t_{m-1}|m-1\rangle .
\end{aligned}
$$

Using these relations, the Hamiltonian Eq. (1) can be cast into a second-quantized form,

$$
\begin{aligned}
\hat{H}= & \sum_{m} \int_{0}^{2 \pi} d \varphi\left[\frac{\left\{\left(i \nabla_{\varphi}-\gamma m\right) \hat{c}_{\varphi, m}^{\dagger}\right\}\left\{\left(-i \nabla_{\varphi}-\gamma m\right) \hat{c}_{\varphi, m}\right\}}{2 M \rho^{2}}\right. \\
& +\alpha\left(t_{m}^{2}+t_{m-1}^{2}\right) \hat{c}_{\varphi, m}^{\dagger} \hat{c}_{\varphi, m}+\alpha\left(t_{m} t_{m+1} \hat{c}_{\varphi, m+2}^{\dagger} \hat{c}_{\varphi, m}+\text { H.c. }\right) \\
& \left.+\beta\left(-i t_{m} \hat{c}_{\varphi, m+1}^{\dagger} \hat{c}_{\varphi, m}+\text { H.c. }\right)\right]
\end{aligned}
$$

where, $\hat{c}_{\varphi, m}^{\dagger}\left(\hat{c}_{\varphi, m}\right)$ creates (annihilates) an excitation in the state indexed by $\varphi$ and $m$. Note that this excitation describes a single effective "particle" that physically corresponds to the occupation of a composite state $|\varphi, m\rangle$, with the electron at angular position $\varphi$ on the ring and the nanomagnet in the spin state $m$.

Reinterpreting both $m$ and $\varphi$ as spatial coordinates, the Hamiltonian Eq. (2) is analogous to that of a single particle on a $2 \mathrm{D}$ cylinder [Fig. 1(b)]. The dimension spanned by $\varphi$ is continuous and periodic $(\varphi+2 \pi=\varphi)$, while that spanned by $m$ is discrete with a finite number of lattice sites, $N=2 s+1$. Under this mapping, the first line in the Hamiltonian Eq. (2) describes the kinetic energy along the $\varphi$ direction, whereas the second and third lines provide the on-site potential energy as well as hoppings (kinetic energy) along the spin $(m)$ direction. Furthermore, the factor of $-\gamma m$ appearing in the kinetic energy in the $\varphi$ direction can be recognized as a vector potential in the Landau gauge, corresponding to a uniform synthetic magnetic field of strength $\gamma$ through the $\varphi$ - $m$ plane. Thus, this hybrid system mimics a particle moving on a cylinder in the presence of a uniform magnetic field.

Relationship to standard quantum hall systems. As we verify below, our model Eq. (2) therefore belongs to the class of 2D quantum Hall systems with energy bands characterized by nontrivial topological Chern numbers. However, unlike most standard quantum Hall Hamiltonians, our system is continuous in one direction and discrete in the other. This is known as a "coupled wire" configuration, as was first introduced theoretically as a tool for constructing fractional quantum Hall states [32]. At a single-particle level, such coupled wire models have also recently become of interest due to experimental proposals for ultracold atoms [33] and, in the context of synthetic dimensions, for coupled optical cavities [19].

Compared to previously studied coupled wire models, however, our system Eq. (2) has unusual nearest- and next-nearest-neighbour hoppings along the spin direction. For example, these hopping amplitudes are nonuniform, meaning that translational invariance is broken even away from the synthetic "edges" of the system at $m= \pm s$. Such hopping anisotropy is often a feature of synthetic dimensions [1,10], and topological properties, such as the existence of chiral edge states, are expected to be robust provided that the anisotropy is sufficiently weak $[1,10]$, as we now also demonstrate here. 
Topological properties. To calculate the bulk topological properties and anticipate the key signatures of the synthetic magnetic field, we first assume that the hopping along the spin direction is uniform, in order to make analytical arguments. To proceed, we introduce the angular momentum quantum number $l_{\varphi}$ around the ring, which is constrained to take only integer values due to the periodicity of $\varphi$. Assuming uniform hopping, $t_{m} \approx t$, the Hamiltonian is

$$
\begin{aligned}
\hat{H} \approx & \sum_{l_{\varphi}, m}\left[\frac{\left(l_{\varphi}-\gamma m\right)^{2}}{2 M \rho^{2}} \hat{c}_{l_{\varphi}, m}^{\dagger} \hat{c}_{l_{\varphi}, m}\right. \\
& \left.+\alpha t^{2} \hat{c}_{l_{\varphi}, m+2}^{\dagger} \hat{c}_{l_{\varphi}, m}-i \beta t \hat{c}_{l_{\varphi}, m+1}^{\dagger} \hat{c}_{l_{\varphi}, m}+\text { H.c. }\right],
\end{aligned}
$$

where $\hat{c}_{l_{\varphi}, m}^{\dagger}$ is the Fourier transform of $\hat{c}_{\varphi, m}^{\dagger}$ along the $\varphi$ direction, and we have omitted a constant energy offset term. This Hamiltonian does not couple operators with different $l_{\varphi}$. We thus have, for a given value of $l_{\varphi}$, a one-dimensional tight-binding Hamiltonian along the spin direction, where the first term in Eq. (3) is a harmonic trapping potential centered at $m=l_{\varphi} / \gamma$ and the other terms are hopping terms. This is reminiscent of the ordinary Landau-level problem, except that the spin direction, $m$, and the momentum, $l_{\varphi}$, are both discrete. Note that, in general, $l_{\varphi} / \gamma$ is not an integer, and thus the center of the trapping potential falls between lattice sites in spin direction. Due to the discreteness of the spin direction, the energy levels are not completely flat, although, practically, as we see below, we observe very flat energy levels for low-lying states in the bulk of the synthetic 2D system. The emergence of these flat Landau levels is a key signature of the synthetic magnetic field.

If we regard the energy levels as a function of $l_{\varphi}$ as energy bands, we can calculate their topological Chern numbers analytically, finding that the Chern number of every band is always equal to one regardless of the values of $\alpha$ and $\beta$ [31]. This behavior agrees with previous studies of coupled wire models [19,32,33], where $\alpha=0$. The nonzero value of the Chern number implies that, from the bulk-boundary correspondence, between the $n$th and $(n+1)$ th bulk energy levels, there are $n$ chiral edge modes at each boundary $m= \pm s$. Physically, these edge modes correspond to spin-dependent chiral currents flowing around the ring; as the largest and smallest spin states serve as the edges of the synthetic spin dimension, the edge-mode chirality means that there are currents mainly involving spin states $m=s$ flowing in one direction around the ring, and currents with $m=-s$ flowing in the opposite direction. This is the second key signature of the synthetic magnetic field.

While we arrived at these expectations assuming uniform hopping $t_{m}=t$, the result is more general. In the original Hamiltonian Eq. (2), the momentum $l_{\varphi}$ is also a good quantum number. As we reintroduce nonuniformity of the hoppings, the bands as a function of $l_{\varphi}$ can become deformed, but as long as band gaps do not close, topological properties, such as the number of chiral edge modes which appear at $m= \pm s$, do not change. Nonetheless, the anisotropy of the full model leaves characteristic fingerprints, as we demonstrate below.

Energy spectrum. To illustrate the topological robustness of the edge states in the full anisotropic model Eq. (2), we

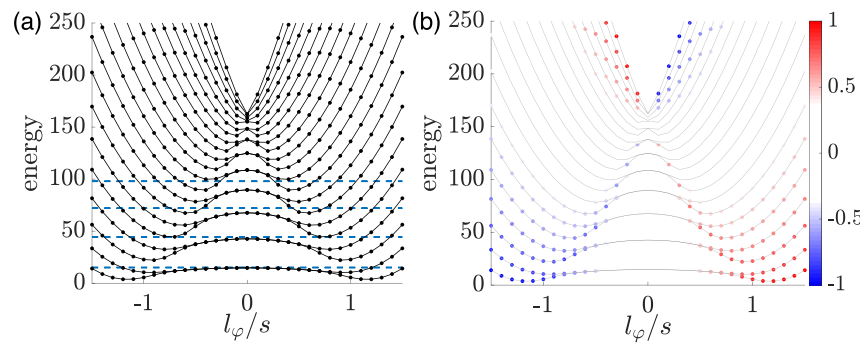

FIG. 2. (a) Energy spectrum as a function of $l_{\varphi} / s$. We used $s=$ $10, \alpha=1.5 /\left(2 M \rho^{2}\right), \beta=0$, and $\gamma=1.2$. Dashed lines are ideal Landau-level energies calculated with $t_{m}=t_{0}$ and $l_{\varphi}=0$. Solid lines are guide to the eyes. (b) The energy spectrum of the same system, with color representing the mean spin of the corresponding states. We observe that states corresponding to dispersive regions have spins concentrated around the edge of the synthetic dimension $(m= \pm s)$, whereas low-lying nondispersive regions have spin concentrated away from the edge in the synthetic dimension. This spin dependence of the energy dispersion shows that we have chiral edge states in the synthetic two-dimensional lattice.

plot the energy spectrum in Fig. 2. Figure 2(a) shows the energy spectrum as a function of $l_{\varphi}$, normalized by the total spin $s=10$ with $\alpha=1.5 /\left(2 M \rho^{2}\right), \beta=0$, and $\gamma=1.2$. The low-lying levels with small $\left|l_{\varphi}\right|$ show weak dispersion; these are almost-flat Landau levels. Since $\beta=0$, states with even $m$ and odd $m$ do not couple, so these levels are nearly twofold degenerate. Plotted in dashed lines are ideal bulk Landau-level energies calculated from Eq. (3), assuming $t_{m}$ to be equal to $t_{0}$ and $l_{\varphi}=0$. They agree well with the energy spectrum for low-lying states, verifying that the introduction of nonuniform hopping $t_{m}$ does not alter the basic phenomenon of bulk Landau levels. Analytical details of the Landau-level spacing and the associated cyclotron frequency are derived in the Supplemental Material [31].

Away from the central region, we see that Landau levels split into two and eventually move up in energy, corresponding to chiral edge states, with states concentrated around $m=$ $s$ propagating in one direction and those concentrated around $m=-s$ propagating in the opposite direction. To clarify the nature of these chiral edge states, we plot the spin-expectation value of these eigenstates $\left\langle S_{z}\right\rangle$ in color in Fig. 2(b). States in red are concentrated on the edge at $m=s$, and states in blue are on the other edge at $m=-s$, showing that the propagating states are edge states in the synthetic dimension. Since each bulk Landau level eventually moves up in energy and becomes an edge state as $\left|l_{\varphi}\right|$ increases, the net number of chiral-edge modes propagating in the same direction is equal to the number of bands below the energy. This observation is in agreement with the fact introduced above that each Landau level has a Chern number of one. We note that at higher energies the propagation direction in the edge states is reversed. This is a finite-size effect, as although the Chern number of each band is one when calculated in the bulk, this assumed an infinite number of bands. For a finite number of $2 s+1$ bands, the Chern numbers must sum to zero, leading to the observed reversal at large energies.

Including a nonzero $\beta$ splits the nearly twofold degeneracy of the Landau levels, but otherwise does not significantly alter 

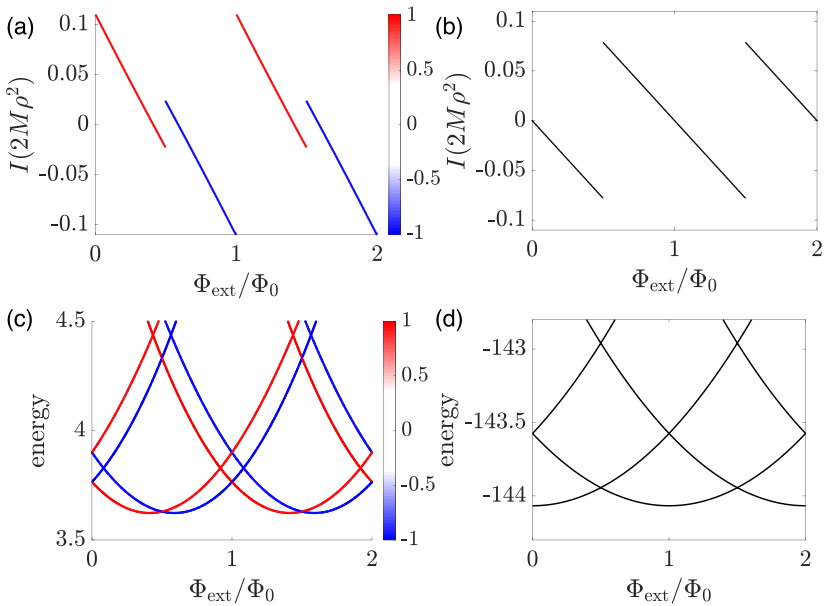

FIG. 3. Persistent current Eq. (5) in the single-particle ground state as a function of the externally controlled flux $\Phi_{\text {ext }}$, for (a) $\alpha=1.5 /\left(2 M \rho^{2}\right)$ and (b) $\alpha=-1.5 /\left(2 M \rho^{2}\right)$, with other parameters as in Fig. 2, where the colors represent the average spin, $\left\langle S_{z}\right\rangle / s$. For both (a) and (b), the persistent current jumps when there is a transition in the lowest energy state as shown in panels (c) and (d), respectively. Notably, when $\alpha>0$ in (a) and (c), the ground state is dominated by the edge modes, and the persistent current exhibits characteristic spin-switching jumps whenever the magnetic flux is increased by a half flux quantum. On the other hand, when $\alpha<0$ in (b) and (d), the ground state is localized in the middle of the synthetic dimension with $\left|\left\langle S_{z}\right\rangle / s\right|<0.1$, and the persistent current only jumps if the magnetic flux is increased by a flux quantum, as is typical also in a quantum ring without a nanomagnet.

the energy spectrum [31]. In particular, the bulk levels remain flat, and each bulk level still leads to one chiral edge mode. The robustness of these edge modes in the energy spectrum reflects their topological nature.

Persistent currents of the single-particle ground state. As established on general grounds above, a key hallmark of our model is the appearance of localized edge modes with respect to the synthetic dimension of spin states. Importantly, the interplay of this physics with the magnet's anisotropy also leads to clear signatures in the persistent current of the singleparticle ground state of the combined system. To illustrate this, we now pierce the ring with an extra externally controlled flux $\Phi_{\text {ext }}$, so Hamiltonian Eq. (1) becomes

$$
H=\frac{1}{2 M \rho^{2}}\left(-i \nabla_{\varphi}-\gamma S_{z}-\frac{\Phi_{\mathrm{ext}}}{\Phi_{0}}\right)^{2}+\alpha S_{x}^{2}+\beta S_{y},
$$

where $\Phi_{0}$ is the magnetic flux quantum. The azimuthal particle velocity is then $\dot{r}_{\varphi}=-(1 / e) \partial H / \partial A_{\varphi}$, where $A_{\varphi}$ is the total magnetic vector potential due to both the nanomagnet and the external flux. This gives an expression for the persistent current around the ring as

$$
I=\frac{\left\langle\dot{r}_{\varphi}\right\rangle}{2 \pi \rho}=\frac{1}{2 \pi M \rho^{2}}\left(l_{\varphi}-\gamma\left\langle S_{z}\right\rangle-\frac{\Phi_{\mathrm{ext}}}{\Phi_{0}}\right),
$$

where the expectation values are taken with respect to the single-particle ground state. The persistent current is shown in Fig. 3(a), for the parameters of Fig. 2, where the colors denote the mean spin of the magnet, indicating that the ground-state persistent current is associated with large spin-polarizations. This can be understood by noting that the lowest energy states in Fig. 2 are modes localized at edges of the spin synthetic dimension. Although the existence of edge modes is a general characteristic of quantum Hall systems, their dominance in the ground state is a new feature due to the magnetic anisotropy; for $\alpha>0$, as in Fig. 3(a), the anisotropy favors extremal spin values, as it generates an inverted harmonic trap $\alpha\left(t_{m}^{2}+\right.$ $\left.t_{m-1}^{2}\right)=\alpha\left[s(s+1)-m^{2}\right]$ in the synthetic dimension Eq. (2) as well as nonuniform hopping terms. Instead, if $\alpha<0$, the ground state and hence the persistent current are localized around the middle of the synthetic dimension, as shown in Fig. 3(b).

Another clear and unusual signature of the edge modes is in the spin-switching jumps of the persistent current in Fig. 3(a), occurring whenever $\Phi_{\text {ext }} / \Phi_{0}=n / 2$, with $n$ being any integer; in Fig. 3(b), by contrast, the persistent current only jumps when $n$ is an odd integer. Physically, these jumps correspond to parameters where the ground state becomes doubly degenerate and then switches between different values of $l_{\varphi}$, as shown in Figs. 3(c) and 3(d), where we plot the lowest energy states for panels (a) and (b), respectively. We provide analytical considerations for the ground-state transitions in the limit $\alpha=\beta=0$ in Ref. [31].

Many-electron case. Let us consider the case where there are $\mathcal{N}$ electrons, each coupled to the same nanomagnet according to Hamiltonian Eq. (4), whilst the electron-electron interactions only depend on the relative position of electrons. The many-body Hamiltonian can then be written as

$$
\begin{aligned}
H_{\mathrm{MB}}= & \frac{1}{2 M_{\mathrm{tot}} \rho^{2}}\left(-i \nabla_{\varphi_{\mathrm{CM}}}-\mathcal{N}\left(\gamma S_{z}+\frac{\Phi_{\mathrm{ext}}}{\Phi_{0}}\right)\right)^{2} \\
& +\alpha S_{x}^{2}+\beta S_{y}+H_{\mathrm{rel}},
\end{aligned}
$$

where $M_{\mathrm{tot}}=\mathcal{N} M$ is the total mass of electrons, $-i \nabla_{\varphi_{\mathrm{CM}}} \equiv$ $-i \nabla_{\varphi_{1}}-i \nabla_{\varphi_{2}} \cdots-i \nabla_{\varphi_{\mathcal{N}}}$ represents their total angular momentum, and $H_{\text {rel }}$ is the Hamiltonian of their relative motion, which is not affected by the presence of the nanomagnet. The important observation is that the nanomagnet only couples to the collective motion of the electrons, whilst the relative motion is completely decoupled. Physically, this means that the characteristic edge states and spin-switching persistentcurrent jumps will now emerge from the center-of-mass motion of electrons around the ring. This should facilitate the experimental observation and interpretation of the described effects, in analogy to what has previously been found for persistent currents [26,27], which can be accurately described in a noninteracting single-particle picture.

In conclusion, we have demonstrated that hybrid nanomagnetic-electronic mesoscopic systems can display topological effects based on the interpretation of the nanomagnet spin as a synthetic dimension. The key signatures are flat Landau-level bands and spin-polarized chiral currents. In the presence of an additional external magnetic flux, the magnet's anisotropy leads to spin-switching behavior in the persistent current of the single-particle ground state. While we have focused on the mesoscopic interpretation of the system, where such a magnet could be, e.g., a molecular magnet [28-30], it is worthwhile mentioning that the components are 
generic and can be realized in other ways, in particular using synthetic quantum engineering. For instance, we can envisage quantum-optical realizations in which the large spin arises from the collective interactions of two-level systems as in a Dicke model [34], the flux is induced via an artificial gauge field [35], and similar atom-optical realizations in which the described effects may serve as robust tools for quantum control [36,37].
Acknowledgments. H.M.P. is supported by the Royal Society via Grants No. UF160112, No. RGFlEAl180121, and No. RGF|R1\180071. T.O. is supported by JSPS KAKENHI Grant No. JP18H05857, RIKEN Incentive Research Project, and the Interdisciplinary Theoretical and Mathematical Sciences Program (iTHEMS) at RIKEN. H.S. acknowledges funding by EPSRC via Grant No. $\mathrm{EP} / \mathrm{P} 010180 / 1$.
[1] A. Celi, P. Massignan, J. Ruseckas, N. Goldman, I. B. Spielman, G. Juzeliūnas, and M. Lewenstein, Synthetic Gauge Fields in Synthetic Dimensions, Phys. Rev. Lett. 112, 043001 (2014).

[2] T. Ozawa and H. M. Price, Topological quantum matter in synthetic dimensions, Nat. Rev. Phys. 1, 349 (2019).

[3] O. Boada, A. Celi, J. I. Latorre, and M. Lewenstein, Quantum Simulation of an Extra Dimension, Phys. Rev. Lett. 108, 133001 (2012).

[4] D. I. Tsomokos, S. Ashhab, and F. Nori, Using superconducting qubit circuits to engineer exotic lattice systems, Phys. Rev. A 82, 052311 (2010).

[5] D. Jukić and H. Buljan, Four-dimensional photonic lattices and discrete tesseract solitons, Phys. Rev. A 87, 013814 (2013).

[6] M. Mancini, G. Pagano, G. Cappellini, L. Livi, M. Rider, J. Catani, C. Sias, P. Zoller, M. Inguscio, M. Dalmonte, and L. Fallani, Observation of chiral edge states with neutral fermions in synthetic Hall ribbons, Science 349, 1510 (2015).

[7] B. K. Stuhl, H. I. Lu, L. M. Aycock, D. Genkina, and I. B. Spielman, Visualizing edge states with an atomic Bose gas in the quantum Hall regime, Science 349, 1514 (2015).

[8] B. Gadway, Atom-optics approach to studying transport phenomena, Phys. Rev. A 92, 043606 (2015).

[9] E. J. Meier, F. A. An and B. Gadway, Atom-optics simulator of lattice transport phenomena, Phys. Rev. A 93, 051602 (2016).

[10] H. M. Price, T. Ozawa, and N. Goldman, Synthetic dimensions for cold atoms from shaking a harmonic trap, Phys. Rev. A 95, 023607 (2017).

[11] L. F. Livi, G. Cappellini, M. Diem, L. Franchi, C. Clivati, M. Frittelli, F. Levi, D. Calonico, J. Catani, M. Inguscio and L. Fallani, Synthetic Dimensions and Spin-Orbit Coupling with an Optical Clock Transition, Phys. Rev. Lett. 117, 220401 (2016).

[12] S. Kolkowitz, S. L. Bromley, T. Bothwell, M. L. Wall, G. E. Marti, A. P. Koller, X. Zhang, A. M. Rey and J. Ye, Spin-orbitcoupled fermions in an optical lattice clock, Nature 542, 66 (2017).

[13] B. Sundar, B. Gadway and K. R. Hazzard, Synthetic dimensions in ultracold polar molecules, Sci. Rep. 8, 3422 (2018).

[14] I. Martin, G. Refael and B. Halperin, Topological Frequency Conversion in Strongly Driven Quantum Systems, Phys. Rev. X 7, 041008 (2017).

[15] M. Schmidt, S. Kessler, V. Peano, O. Painter, and F. Marquardt, Optomechanical creation of magnetic fields for photons on a lattice, Optica 2, 635 (2015).

[16] X.-W. Luo, J.-S. Xu, G.-C. Guo, X. Zhou, C.-F. Li, and Z.-W. Zhou, Quantum simulation of 2D topological physics in a 1D array of optical cavities, Nat. Commun. 6, 7704 (2015).

[17] T. Ozawa, H. M. Price, N. Goldman, O. Zilberberg, and I. Carusotto, Synthetic dimensions in integrated photonics: From optical isolation to four-dimensional quantum Hall physics, Phys. Rev. A 93, 043827 (2016).

[18] L. Yuan, Y. Shi, and S. Fan, Photonic gauge potential in a system with a synthetic frequency dimension, Opt. Lett. 41, 741 (2016).

[19] T. Ozawa and I. Carusotto, Synthetic Dimensions with Magnetic Fields and Local Interactions in Photonic Lattices, Phys. Rev. Lett. 118, 013601 (2017).

[20] F. Cardano, A. D’Errico, A. Dauphin, M. Maffei, B. Piccirillo, C. de Lisio, G. De Filippis, V. Cataudella, E. Santamato, L. Marrucci, M. Lewenstein, and P. Massignan, Detection of Zak phases and topological invariants in a chiral quantum walk of twisted photons, Nat. Commun. 8, 15516 (2017).

[21] L. Yuan, Q. Lin, M. Xiao, and S. Fan, Synthetic dimension in photonics, Optica 5, 1396 (2018).

[22] E. Lustig, S. Weimann, Y. Plotnik, Y. Lumer, M. A. Bandres, A. Szameit, and M. Segev, Photonic topological insulator in synthetic dimensions, Nature 567, 356 (2019).

[23] C. W. Peterson, W. A. Benalcazar, M. Lin, T. L. Hughes, and G. Bahl, Strong Nonreciprocity in Modulated Resonator Chains through Synthetic Electric and Magnetic Fields, Phys. Rev. Lett. 123, 063901 (2019).

[24] A. Dutt, M. Minkov, Q. Lin, L. Yuan, D. A. B. Miller, and S. Fan, Experimental band structure spectroscopy along a synthetic dimension, Nat. Commun. 10, 3122 (2019).

[25] M. Büttiker, Y. Imry, and R. Landauer, Josephson behavior in small normal one-dimensional rings, Phys. Lett. A 96, 365 (1983).

[26] H. Bluhm, N. C. Koshnick, J. A. Bert, M. E. Huber, and K. A Moler, Persistent Currents in Normal Metal Rings, Phys. Rev. Lett. 102, 136802 (2009).

[27] A. C. Bleszynski-Jayich, W. E. Shanks, B. Peaudecerf, E. Ginossar, F. von Oppen, L. Glazman, and J. G. E. Harris, Persistent currents in normal metal rings, Science 326, 272 (2009).

[28] R. Sessoli, D. Gatteschi, A. Caneschi, and M. A. Novak, Magnetic bistability in a metal-ion cluster, Nature 365, 141 (1993).

[29] C. Calero, E. M. Chudnovsky, and D. A. Garanin, Quantum dynamics of a nanomagnet in a rotating field, Phys. Rev. B 72 , 024409 (2005). 
[30] J. Tejada, R. D. Zysler, E. Molins, and E. M. Chudnovsky, Evidence for Quantization of Mechanical Rotation of Magnetic Nanoparticles, Phys. Rev. Lett. 104, 027202 (2010).

[31] See Supplemental Material at http://link.aps.org/supplemental/ 10.1103/PhysRevResearch.2.032017 for detailed discussions of the Chern number, preferred spin orientations, and ground-state transitions.

[32] C. L. Kane, R. Mukhopadhyay, and T. C. Lubensky, Fractional Quantum Hall Effect in an Array of Quantum Wires, Phys. Rev. Lett. 88, 036401 (2002).

[33] J. C. Budich, A. Elben, M. Łącki, A. Sterdyniak, M. A. Baranov, and P. Zoller, Coupled atomic wires in a synthetic magnetic field, Phys. Rev. A 95, 043632 (2017).
[34] B. M. Garraway, The Dicke model in quantum optics: Dicke model revisited, Phil. Trans. R. Soc. A 369, 1137-1155 (2011).

[35] T. Ozawa, H. M. Price, A. Amo, N. Goldman, M. Hafezi, L. Lu, M. C. Rechtsman, D. Schuster, J. Simon, O. Zilberberg, and I. Carusotto, Topological photonics, Rev. Mod. Phys. 91, 015006 (2019).

[36] N. Goldman, G. Juzeliūnas, P. Öhberg, and I. B. Spielman, Light-induced gauge fields for ultracold atoms, Rep. Prog. Phys. 77, 126401 (2014).

[37] N. R. Cooper, J. Dalibard, and I. B. Spielman, Topological bands for ultracold atoms, Rev. Mod. Phys. 91, 015005 (2019). 